\title{
Araştırma Makalesi / Research Article \\ İçi Kovuk Metal Oksit Kürelerin Hazırlanmasında Blok Kopolimer İle Stabilize Edilmiş Polistiren Mikrokürelerin Şablon Olarak Kullanımı
}

\author{
Gökhan KOÇAK ${ }^{1}$ \\ ${ }^{1}$ Adıyaman Üniversitesi, Teknik Bilimler Meslek Yüksekokulu, Kimya ve Kimyasal Işsleme Teknolojileri Bölümü, \\ Adıyaman.
}

e-posta: gkocak@adiyaman.edu.tr. ORCID ID: http://orcid.org/0000-0002-1169-6934

\begin{abstract}
Öz
Bu çalışmada, çift tabakalı mangan oksit@nikel oksit içi kovuk kürelerin üretiminde şablon olarak poli[2(dietilamino)etil metakrilat-blok-2-(dimetilamino)etil metakrilat] (PDEA-b-PDMA) diblok kopolimer ile

Anahtar Kelimeler

Blok kopolimer; emülsiyon;

Polimerizasyonu; metal oksit;

içi kovuk küre; nikel oksit stabilize edilmiş polistiren (PS) mikroküreleri kullanıldı. Emülsiyon polimerizasyonu ile blok kopolimer ile stabilize edilmiş eş dağılımlı PS mikroküreler sert şablon olarak kullanılarak sırasıyla $\mathrm{KMnO}_{4}$ ve $\mathrm{NiSO}_{4}$ 'ün kontrollü çöktürme yöntemiyle metal oksit öncüsü bileşikler ile kaplandı. Kalsinasyon işlemi ile mangan oksit@nikel oksit içi kovuk çift tabakalı metal oksit küreler üretildi. PS küreler ve içi kovuk metal küreler dinamik ışık saçılması (DLS), termogravimetrik analiz (TGA), ışık mikroskobu, X-ışını kırınımı (XRD) ve taramalı elektron mikroskobu (SEM) ile karakterize edildi. Mangan oksit@nikel oksit içi kovuk kürelerin, iç tabakasında kalan mangan oksit tabakasının ağırlıkça oranı oldukça düşükken ( \%4), dış tabakasında yer alan nikel oksit tabakasının ağırlıkça oldukça yüksektir ( \%96). Üretilen metal oksit kürelerin 1,60$1,77 \mu \mathrm{m}$ çapında olduğu ve bu kürelerin kabuk kalınlığının ise $200 \mathrm{~nm}$ civarındadır. İçi kovuk kürelerin dış tabakası, kataliz çalışmaları için önemli avantajlar sunan oldukça pürüzlü bir dış yüzeye sahip olduğu tespit edildi.
\end{abstract}

\section{Use of Block Copolymer Stabilized Polystyrene Microspheres as Templates for Preparation of Hollow Metal Oxide Spheres}

\footnotetext{
Abstract

In this study, poly[2-(diethylamino) ethyl methacrylate-block-2-(dimethylamino) ethyl methacrylate] (PDEA-b-PDMA) diblock copolymer stabilized polystyrene (PS) microspheres were used as a template in the production of double layer manganese oxide@nickel oxide hollow spheres. Monodispers PS

Keywords

Block copolymer; emulsion;

polymerization; metal oxide; hollow sphere; nickel oxide microspheres stabilized with block copolymer by emulsion polymerization were coated with metal oxide precursors using the controlled precipitation method of $\mathrm{KMnO}_{4}$ and $\mathrm{NiSO}_{4}$, respectively. Double layer manganese oxide@nickel oxide hollow spheres were produced by calcination process. PS spheres and hollow metal spheres were characterized by dynamic light scattering (DLS), thermogravimetric analysis (TGA), light microscope, X-ray diffraction (XRD) and scanning electron microscope (SEM). The weight of the manganese oxide@nickel oxide hollow spheres in the inner layer of the manganese oxide is quite low ( $4 \%$ ), while the outer layer of the nickel oxide layer is quite high ( 96\%). The produced metal oxide spheres have a diameter of 1.60-1.77 $\mu \mathrm{m}$ and the shell thickness of the spheres is around $200 \mathrm{~nm}$. The outer layer of hollow spheres was found to have a rather rough outer surface, which offers significant advantages for catalysis studies.
} 


\section{Giriş}

Nano ve mikro boyutlu metal oksitler özellikle kataliz uygulamalar başta olmak üzere oldukça popüler malzemelerden biridir (Gawande et al. 2012, Pal and Pal 2015). Özel bir malzeme sınıfı olan içi kovuk yapılar; geniş yüzey alanı, düşük yoğunluk, bol miktardaki iç boşluk ve diğer optik, manyetik ve katalitik özellikleri ile dış kabuğun içeriğine göre birçok uygulama için iyi bir adaydır (Hu et al. 2011, X. J. Wang et al. 2016). Bu özel yapıların içi boş olmasından dolayı, yüzey alanı belirgin şekilde daha büyükken, yoğunluk aynı bileşim ve büyüklükte olan benzerlerinden çok daha düşüktür. $\mathrm{Bu}$ özellikleri kataliz uygulamalarında önemli avantaj sağlar (X. J. Wang et al. 2016). Nano ve mikro boyutlardaki içi kovuk yapılar; küre, tüp, lif ve kutu gibi şekillerde olabilmektedir. Dış kabuk sayısına göre ise; tek, çift ve çok içi kovuk yapılar olarak adlandırılırlar (Hu et al. 2011, X. J. Wang et al. 2016). İçi kovuk kürelerin istenilen boyutta, eş boyut dağılımlı, tekrarlanabilir ve uygun maliyetle üretilmesi pratik uygulamalar için önemlidir. Sert şablon (karbon, silika, polimer, seramik, inorganik küreler vb.), yumuşak şablon (yüzey aktif madde, blok kopolimer, gaz kabarcığı, bakteriler, emülsiyon damlası) ve kendi-kendine şablon gibi farklı yaklaşımlar kullanılarak içi kovuk küreler üretilmektedir (Hu et al. 2011, X. J. Wang et al. 2016). Bu teknikler arasında sert şablonların (polimer, silika ve karbon) kullanımı kavramsal olarak en basit olanıdır (Hu et al. 2011, Sun et al. 2006, X. J. Wang et al. 2016). Polistiren (PS) ve türevleri ve poli(metil metakrilat) (PMMA) kolay ve düşük maliyetleri sebebi ile sıklıkla polimerik sert şablon olarak kullanılmaktadır (X. J. Wang et al. 2016).

Sert şablon olarak kullanılan PS kürelerin yüzey yükü; emülsiyon polarizasyonunda kullanılan radikal başlatıcının türüne (Watanabe et al. 2007), kullanılan stabilizatör ajanına (Amalvy et al. 2004, Bousquet et al. 2010, Itoh et al. 2014, MunozBonilla et al. 2010, Reis et al. 2010) ve çeşitli kimyasallarla muamele edilmesiyle ayarlanabilir (Amari et al. 2017, Cai et al. 2014, Deng et al. 2008). Diğer bir polimerik sert şablon yaklaşımda ise çeşitli yüzeylerden (polimerik ve silika) polimerizasyon ile üretilen küresel fırçalardır (Huang et al. 2012, Lu et al. 2009, Nie et al. 2016, Polzer et al. 2013, Zhu et al. 2012). Küresel fırçalar çoğunlukla nano boyutlu inorganik partiküllerin üretiminde kullanılmıştır. Şablon malzemelerin yüzeyinin inorganik türler ile kaplanmasında; (i) tabaka tabaka kaplama (LbL), (ii) adsorpsiyon ve (iii) kontrollü çöktürme gibi metotlar kullanılmaktadır (Hu et al. 2011, X. J. Wang et al. 2016). Daha sonra polimer, uygun çözücüde çözülerek veya kalsinasyon ile yakılarak içi kovuk metal oksit küreler elde edilmektedir (Hu et al. 2011, X. J. Wang et al. 2016). Bu çalışmada kullanılan kontrollü çöktürme tekniği ile $\mathrm{Co}_{3} \mathrm{O}_{4}$ (Ohnishi et al. 2006), $\mathrm{Fe}_{3} \mathrm{O}_{4}$ (Ohnishi et al. 2006, Ye et al. 2010), $\alpha$ $\mathrm{Fe}_{2} \mathrm{O}_{3}$ (Ohnishi et al. 2006, Qian et al. 2007, Ye et al. 2010), NiO (Qian et al. 2007, Tong et al. 2012, D. B. Wang et al. 2005, Wu et al. 2007, Zhang et al. 2012), ZnO (Qian et al. 2007, Yang et al. 2016), CuO (Kawahashi and Shiho 2000, Qian et al. 2007), $\mathrm{Ga}_{2} \mathrm{O}_{3}$ (Qian et al. 2007), $\mathrm{MnO}_{2}$ (Fei et al. 2008, Li et al. 2006), $\mathrm{ZrO}_{2}$ (Kawahashi et al. 1991), $\mathrm{CoFe}_{2} \mathrm{O}_{4}$ (Yoon 2014) gibi içi kovuk metal oksit küreler farklı uygulamalarda kullanılmak üzere üretilmiştir.

Blok kopolimerlerle stabilize edilmiş polimerik küreler; polimer bilimciler tarafından daha monodispers veya çevre duyarlı küresel partiküllerin üretilmesinde tercih edilmişlerdir (Amalvy et al. 2004, Bousquet et al. 2010, Itoh et al. 2014, Munoz-Bonilla et al. 2010, Reis et al. 2010). Elde edilen küresel partiküller, sıcaklık ve pH gibi dış etkilerle şişme-büzülme davranışı veya yüzey özelliklerinde değişmeler sergilerler (Amalvy et al. 2004, Bousquet et al. 2010, Itoh et al. 2014, Munoz-Bonilla et al. 2010, Reis et al. 2010). Bu polimerik yapılar, fırça polimerler gibi çevresinde polimer saçaklar barındırsalar da fırça polimerlere göre oldukça basit şekilde üretilirler. Diğer bir taraftan bok kopolimerlerle stabilize edilmiş polimerik küreler, yüzeyi fonksiyonel hale getirilmiş PS kürelere göre daha fazla fonksiyonel gruba sahiptir. Fonksiyonel gruplarca zengin yapı hem adsorpsiyon hem de inorganik türlerin yüzeyde çekirdekleşmesi için uygun ortam sağlar (Huang et 
al. 2012, Lu et al. 2009, Nie et al. 2016, Polzer et al. 2013, Zhu et al. 2012).

Bu çalışmada, blok kopolimerlerle stabilize edilmiş PS küreler içi kovuk küresel metal oksit üretilmesinde sert şablon olarak kullanılması amaçlanmıştır. PS küreler tersiyer amin içerikli bloklardan oluşan poli[2-(dietilamino)etil metakrilat-blok-2-(dimetilamino)etil metakrilat] (PDEA-b-PDMA) diblok kopolimeri kullanılarak emülsiyon polimerizasyonu ile üretildi. PS kürelerin etrafında yer alan PDMA saçakları metal oksit öncüsü tuzun çekirdekleşmesi ve sonrasında PS kürelerin bu öncü tuzlarla kaplanması için fonksiyonel ortam sağlamıştır. Adsorsiyon ve kontrollü çöktürme teknikleri ile yüzeyde metal oksit öncüleri toplanmış ve kalsinasyon işlemi ile hem polimerik kısım yok edilmiş hem de kristalin mangan oksit@nikel oksit içi kovuk küreler üretilmiştir. Daha basit ve çeşitli fonksiyonellikte üretilebilecek blok kopolimerle stabilize edilmiş PS küreler, farklı türde içi kovuk metal oksit kürelerin üretilmesinde yeni bir model olma adayıdır.

\section{Materyal ve Metot}

Monomer olarak stiren (Merck), radikal başlatıcı olarak 2,2'-azodiisobutironitrile (AIBN, Acros), çözücü olarak methanol $(\mathrm{MeOH})$ ve destile su karışımı, ve stabilizatör olarak PDEA- $b$-PDMA diblok kopolimeri küresel PS kürelerin emülsiyon polimerizasyonunda üretilmesinde kullanıldı (Bütün et al. 2004). ilk tabaka elde edilmesinde $\mathrm{KMnO}_{4}$ (Merck), ikinci tabakada ise $\mathrm{NiSO}_{4} 6 \mathrm{H}_{2} \mathrm{O}$ (SigmaAldrich, SI-AL), ve polivinilpirolidon (PVP, Sigma, $40000 \mathrm{~g} / \mathrm{mol}$ ) kullanıldı. İkinci tabakada kontrollü çöktürme amacı ile üre (SI-AL) küresel inorganikpolimer hibrit malzemelerin üretilmesinde kullanıldı. Stiren monomeri bazik alumina (SI-AL) kolondan geçirilerek saflaştırıldı.

PS kürelerin hidrodinamik çapı $\left(R_{h}\right)$ ve polidispersite indeks değerleri (PDI veya $\mu_{2} / \Gamma^{2}$ ) Dinamik ışık saçılması (DLS) ile belirlenmiştir. DLS çalışmaları ALV/CGS-3 kompakt goniometre sistemi (Malvern, Inc, UK) kullanılarak gerçekleştirilmiştir. Bu goniometre sistemi, $\lambda_{0} 632,8 \mathrm{~nm}$ 'de $22 \mathrm{~mW}$ He-Ne lazeri, yüksek kuantum verimliliği ile çalışan fotodiyot dedektörü, bir ALV/LSE-5003 çoklu tau dijital korelatör elektronik sistemi ile donatılmıştır. Bütün ölçümler polimer dispersiyonları üzerinden $90^{\circ}$ sabit açılı saçılmalar ile yapıldı. Veriler ikinci dereceden kümülatif analizlerle değerlendirilmiştir. Çözelti sıcaklığı ısı kontrollü su banyosu ile $\pm 1{ }^{\circ} \mathrm{C}$ duyarlılıkta sabit tutulmuştur. Hazırlanan içi kovuk metal oksit kürelerin polimer-metal oksit'in \% (w/w) oranı termogravimetrik analiz (TGA) cihazı (Seiko SII Extar 6000 TG/DTA) ile yapıldı. Ölçümler farklı ısıtma hızında $\left(1-10^{\circ} \mathrm{C} / \mathrm{min}\right)$ ve $2 \mathrm{~mL} / \mathrm{min}$ kuru hava atmosferinde gerçekleştirildi. Hazırlanan içi kovuk metal oksit kürelerin morfolojileri Işık mikroskobu (Leica DM750) ve Tarmalı elektron mikroskobu (SEM, JEOL JSM 5600LV) ile incelendi. Hazırlanan içi kovuk metal oksit kürelerin toz kırınım desenleri X-Işını Kırınımı (toz-XRD, Panalytical Empyrean difraktometresi) analizi Cu K$\alpha$ radyasyon $(\lambda 1 / 41.54 \AA$ ) kullanılarak, $2 \theta$ açısı 1-60 aralığında ölçüm alınmıştır. Toz kırınım desenleri High Score Plus yazılımında incelenerek pik tayinleri yapılmış, ICDD PDF4+ kütüphanesinden araştırılarak bulunan referans fazları ile de numunenin faz içeriği aydınlatılmıştır.

\subsection{Polistiren (PS) mikro kürelerin emülsiyon polimerizasyonu ile üretilmesi}

Daha önce Grup transfer polimerizasyon (GTP) tekniği ile Bütün vd. (2004) tarafından üretilen PDEA $_{29}-b-$ PDMA $_{68}(0,60 \mathrm{~g}, 16062 \mathrm{~g} / \mathrm{mol}, \mathrm{PDI}: 1,06)$ diblok kopolimer stabilizatörü, AIBN (0,045 g), $5 \mathrm{~mL}$ stiren, $\mathrm{H}_{2} \mathrm{O} / \mathrm{MeOH}(1 / 9,50 \mathrm{~mL})$ çözücü karşımı $1000 \mathrm{rpm}$ hızında azot altında karıştırıldı ve 60 ${ }^{\circ} \mathrm{C}$ 'de reaksiyon sürdürüldü (Şema 1 ). Bir gece reaksiyon sürdürüldükten sonra 10 dakika boyunca 10000 rpm de birkaç kez santrifüj yapılarak ürün çöktürüldü (Şema 1). Elde edilen PS küreler vakumda bir gece boyunca kurutuldu.

\section{2. İ̧i kovuk metal oksit kürelerin üretilmesi}

İçi kovuk metal oksit kürelerin hazırlanma metodu Şema 1'de özetlenmiştir. Blok kopolimer ile stabilize edilen PS kürelerin metal oksit öncüleri ile kaplanması daha önceki çalışmalara oldukça benzer şekilde yapılmıştır (Abdullah et al. 2018, Polzer et 
al. 2013, Qian et al. 2007, Wu et al. 2007). İlk tabaka için özetle, PS küreler $(0,1 \mathrm{~g})$ ilk olarak 10 $\mathrm{mL}$ suda dağıtıldı ve $\mathrm{KMnO}_{4}$ çözeltisi $(0,2 \mathrm{M}, 5 \mathrm{~mL})$ eklenerek bir gece boyunca oda sıcaklığında karıştırıldı (pH 7.2). Reaksiyon sonunda kaplanmış kürelerin renginin açık kahverengi olduğu ve çözeltinin $\mathrm{KMnO}_{4}{ }^{\prime}$ ün rengine sahip olduğu gözlemlendi. Elde edilen malzeme $100{ }^{\circ} \mathrm{C}^{\prime}$ de bir gece kurutuldu.

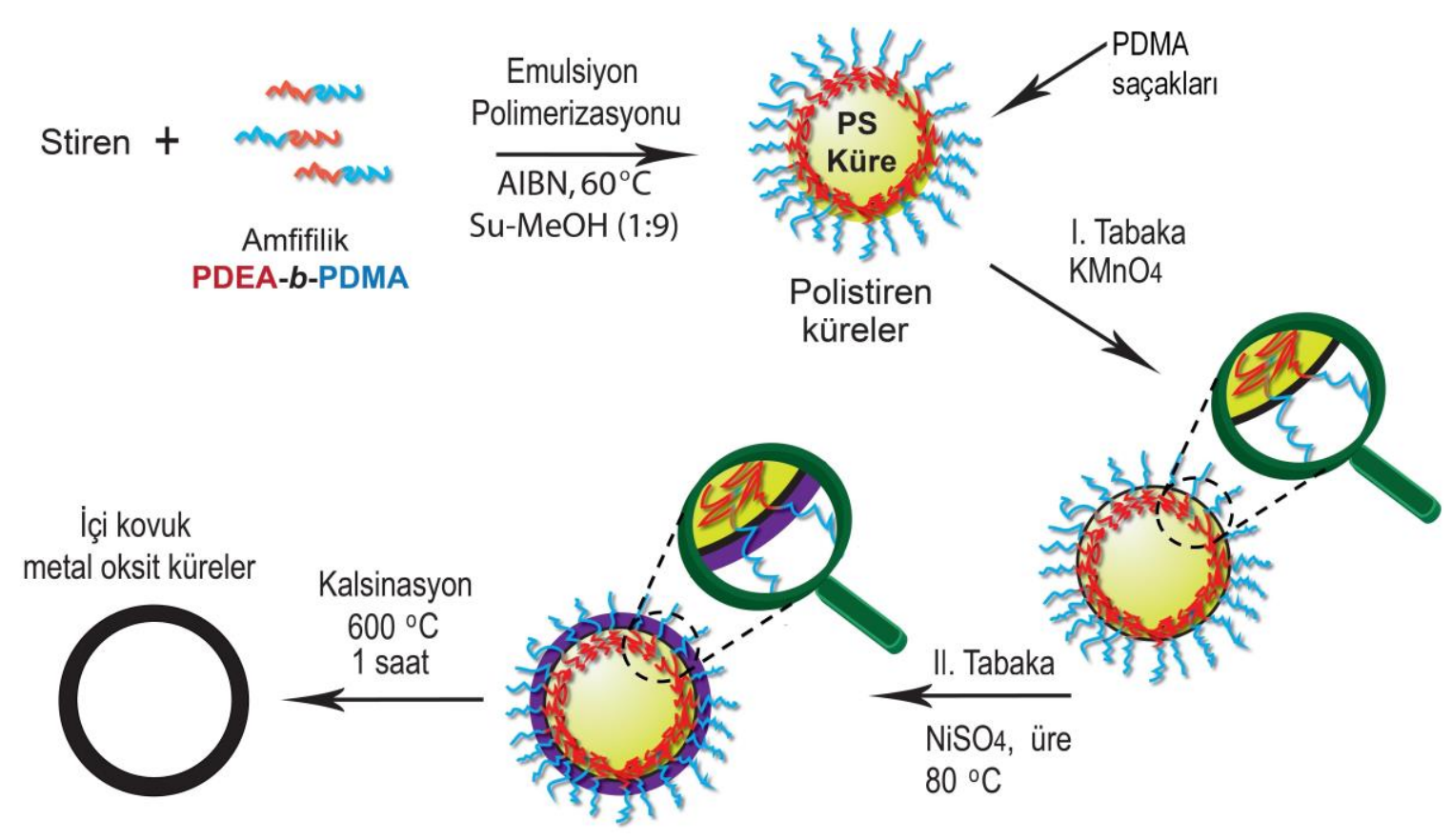

Şema 1. PS mikrokürelerin ve içi kovuk metal oksit kürelerin üretimi.

Son aşamada ise, PS-inorganik hibrit küreler 600 ${ }^{\circ} C^{\prime}$ ye kadar farklı ısıtma programları kullanılarak kuru hava atmosferinde ( $2 \mathrm{~mL} / \mathrm{min}$ ) kalsine edildi. TGA verilerinde $250-285{ }^{\circ} \mathrm{C}$ sonrasında şiddetli yanmaya bağlı olarak TGA kromotogramlarında sapma gözlemlendiğinden bu sıcaklık aralığında ve sonrasında düşük ısıtma hızı $\left(3-5^{\circ} \mathrm{C} / \mathrm{min}\right)$ kullanıldı ve $285^{\circ}{ }^{\circ}$ 'de 60 dakika kadar beklenerek polimerik kısım kontrollü şekilde yakılarak tamamen uzaklaştırıldı. $600{ }^{\circ} \mathrm{C}^{\prime}$ ye gelindikten sonra 1 saat daha ısıtma sürdürülerek kalsinasyon işlemi tamamlandı. Hem TGA hem de sentezlerde uygulanan ısıtma programı Şekil 1'de verilmiştir. Her basamaktaki metal oksit-polimer yüzde oranı TGA ölçümleri ile tespit edildi. XRD ile oluşan metal oksit türü ve oranları belirlendi. Ayrıca, ışık mikroskobu ve SEM ölçümleri ile PS küreler ve oluşan metal oksit kürelerin morfolojisi aydınlatıldı.
İkinci tabaka ise, $\mathrm{KMnO}_{4}$ ile muamele edilen PS küreler $(0,1 \mathrm{~g}) 100 \mathrm{~mL}$ destile su, $\mathrm{NiSO}_{4}(0,2 \mathrm{M}, 3$ $\mathrm{mL})$ ve PVP $(0,15 \mathrm{~g}, 40000 \mathrm{~g} / \mathrm{mol}) 48$ saat boyunca karıştırıldı. Daha sonra $3,4 \mathrm{~g}$ üre suda $(20 \mathrm{~mL})$ çözülerek reaksiyon ortamına eklendi ve $80^{\circ} \mathrm{C}$ ' de 48 saat karıştırıldı. Ürün 5000 rpm'de santrifüjlendi ve birkaç kez destile su ile yıkandı. Santrifüj yapılan çözelti kısmının tamamen berrak olduğu yani tüm öncü tuzun kontrollü şekilde çöktüğü görüldü. Elde edilen malzeme $100{ }^{\circ} \mathrm{C}^{\prime}$ de bir gece kurutuldu. 
ise tıpkı küresel fırçalarda olduğu gibi PDMA bloğuna ait polimer zincir saçaklarının şeklinde varlığını sürdürmüştür. Daha önce yapılan çalışmalardan blok kopolimerin PS küre yapısıyla bütünleşmiş olduğu ve kolayca yapıdan ayrılmadığı bilinmektedir (Amalvy et al. 2004, Bousquet et al. 2010, Itoh et al. 2014, Munoz-Bonilla et al. 2010, Reis et al. 2010). Elde edilen saçaklı PS küreleri etrafındaki PDMA zincirleri daha yüksek oranda metal oksit öncüsü bileşiklerin adsorplanması ve çekirdekleşmesi için daha uygun fonksiyonel gruplara sahiptir. Küresel fırçaların bu özelliğinden yola çıkılarak nanometallerin bu saçaklar bünyesinde üretildiği ve stabilize edilmesini içeren bir çok çalışma yapılmıştır (Huang et al. 2012, Lu et al. 2009, Nie et al. 2016, Polzer et al. 2013, Zhu et al. 2012).

\section{2. İçi kovuk metal oksit kürelerin üretilmesi}

PS küreler ile $\mathrm{KMnO}_{4}^{\prime}$ ın bir gece karıştırıldıktan sonra açık kahverengiye dönmüşken, reaksiyon ortamında hala $\mathrm{KMnO}_{4}{ }^{\prime} \mathrm{e}$ ait olan mor renk mevcuttur. PS kürelerdeki ren değişimi PS küre$\mathrm{KMnO}_{4}$ arasında gerçekleşen redoks reaksiyonu ile oluşan mangan dioksit $\left(\mathrm{MnO}_{2}\right)$ oluşumuna dayandırılabilir. Özellikle polistiren'nin bu türde etkisi olduğu daha önce yapılan çalışmalarda ele alınmış ve stiren- $\mathrm{MnO}_{4}^{-}$arasında gerçekleşen reaksiyon için bir reaksiyon mekanizması da önerilmiştir (Abdullah et al. 2018, Han et al. 2007). Diğer bir çalışmada ise, katyonik polimer saçakları arasında yer alan yüksek konsantrasyondaki $\mathrm{OH}^{-}$ iyonlarının bu indirgenmede rol almasıdır ve bu çalışmadaki muhtemel indirgenme reaksiyonunu izah edebilmektedir (Polzer et al. 2013). Muhtemelen bu iki mekanizma ile çok düşük miktarda da olsa PS kürelerinin yüzeyinde birikmiş ve birinci metal oksit tabakası oluşmuştur. íkinci tabakadaki nikel oksit'in biriktirilmesinde ise $\mathrm{NiSO}_{4}^{\prime}$ 'ın çözünmez tuzunun yavaşça çöktürülmesine dayanmaktadır. Ortama eklenen üre, $80^{\circ} \mathrm{C}$ sıcaklıkta yavaşşa bozunarak $\mathrm{NH}_{3}$ oluştur ve $\mathrm{NiSO}_{4}$ ile $\mathrm{NH}_{3}$ 'ün reaksiyonuyla $\mathrm{PS}$ yüzeyindeki PDMA saçaklarında $\mathrm{Ni}(\mathrm{OH})_{2}$ kontrollü şeklinde çökmeye başlar. Çöktürülen $\mathrm{Ni}(\mathrm{OH})_{2}$ daha sonra termal bozunma (kalsinasyon) ile nikel oksit'e dönüştürülür (Qian et al. 2007, Tong et al. 2012, D. B. Wang et al. 2005, Wu et al. 2007, Zhang et al. 2012). $\mathrm{Ni}(\mathrm{OH})_{2}$ eldesi ve kalsinasyonun için önerilen reaksiyonlar basamaklar halinde aşağıda verilmiştir (Qian et al. 2007, Tong et al. 2012). Nikel tuzu, üre ve PS miktarı, sıcaklık gibi değişkenler PS yüzeyindeki kontrollü çökmeyi önemli derecede etkilediği daha önce yapılan çalışmalarda detaylıca çalışılmıştır (Qian et al. 2007, Tong et al. 2012, D. B. Wang et al. 2005, Wu et al. 2007, Zhang et al. 2012). Çalışmamızda kullanılan $\mathrm{PS}$ küre, $\mathrm{NiSO}_{4}$ ve üre oranı önceki çalışmalardan faydalanarak belirlenmiş ve optimizasyon çalışmaları için uzun süreler harcanmadan homojen ve tamamen küreler kaplanmıştır. Bu faktörlerdeki değişim ve oluşacak yapılar farklı araştırma konusu olabilecek kadar önemlidir. Üre kullanılarak çeşitli metal hidroksitlerin kontrollü çöktürülmesine dayanan bu yöntemle birçok içi kovuk metal oksit kürelerin elde edilmesi mümkündür. Işık mikroskobu ve SEM görüntülerinde de görüleceği gibi PS kürelerin yüzeyinde oldukça homojen birikme sağlanmıştır [Şekil 2(c-f)].

$$
\begin{aligned}
\mathrm{CO}\left(\mathrm{NH}_{2}\right)_{2}+\mathrm{H}_{2} \mathrm{O} & \rightleftharpoons \mathrm{CO}_{2}+2 \mathrm{NH}_{3} \\
\mathrm{NH}_{3}+\mathrm{H}_{2} \mathrm{O} & \rightleftharpoons \mathrm{NH}_{4}^{+}+\mathrm{OH}^{-} \\
\mathrm{M}^{n+}+n \mathrm{OH}^{-} & \longrightarrow \mathrm{M}(\mathrm{OH})_{n} \\
2 \mathrm{M}(\mathrm{OH})_{n} & \longrightarrow \mathrm{M}_{2} \mathrm{O}_{n}+n \mathrm{H}_{2} \mathrm{O}
\end{aligned}
$$

Birinci metal oksit tabasının oluşturulması sonrasında gözlemlenen açık kahverengi PS küreler, birinci tabakayı oluşturan mangan oksit türünün yüzeyde azda olsa biriktiğini göstermektedir. TGA verilerine göre kütlece $600{ }^{\circ} \mathrm{C}$ 'de $\% 1.56$ gibi düşük bir değerde kalıntı mevcut. İkinci kaplamadan sonra TGA verilerine göre $600{ }^{\circ} \mathrm{C}$ 'de kütlece 40,17 oranında metal oksit olduğu görülmektedir (Şekil 1). Buna göre toplam polimer-metal oksit kütlesinin \% 38,61'i nikel oksit içeriğinin olduğunu göstermektedir. Toplam metal oksit kütlesi içindeki nikel oksit'in yaklaşık \%96,1 (w/w) oranında olduğu TGA ölçümleri sonucunda belirlendi. 


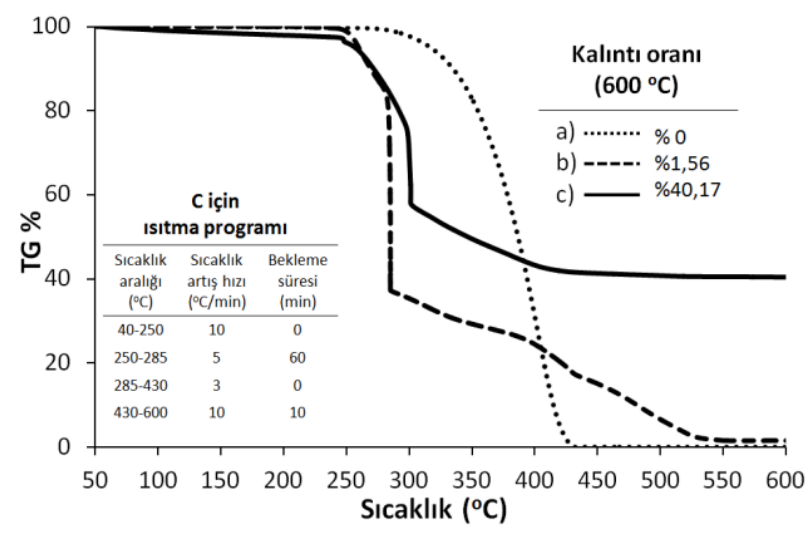

Şekil 1. TGA kromotogramları; PS küreler (a), $\mathrm{KMnO}_{4}$ muamelesi (I. tabaka) sonrası PS küreler (b) ve $\mathrm{NiSO}_{4}+$ üre muamelesi sonrası (II. tabaka) PS küreler (c).
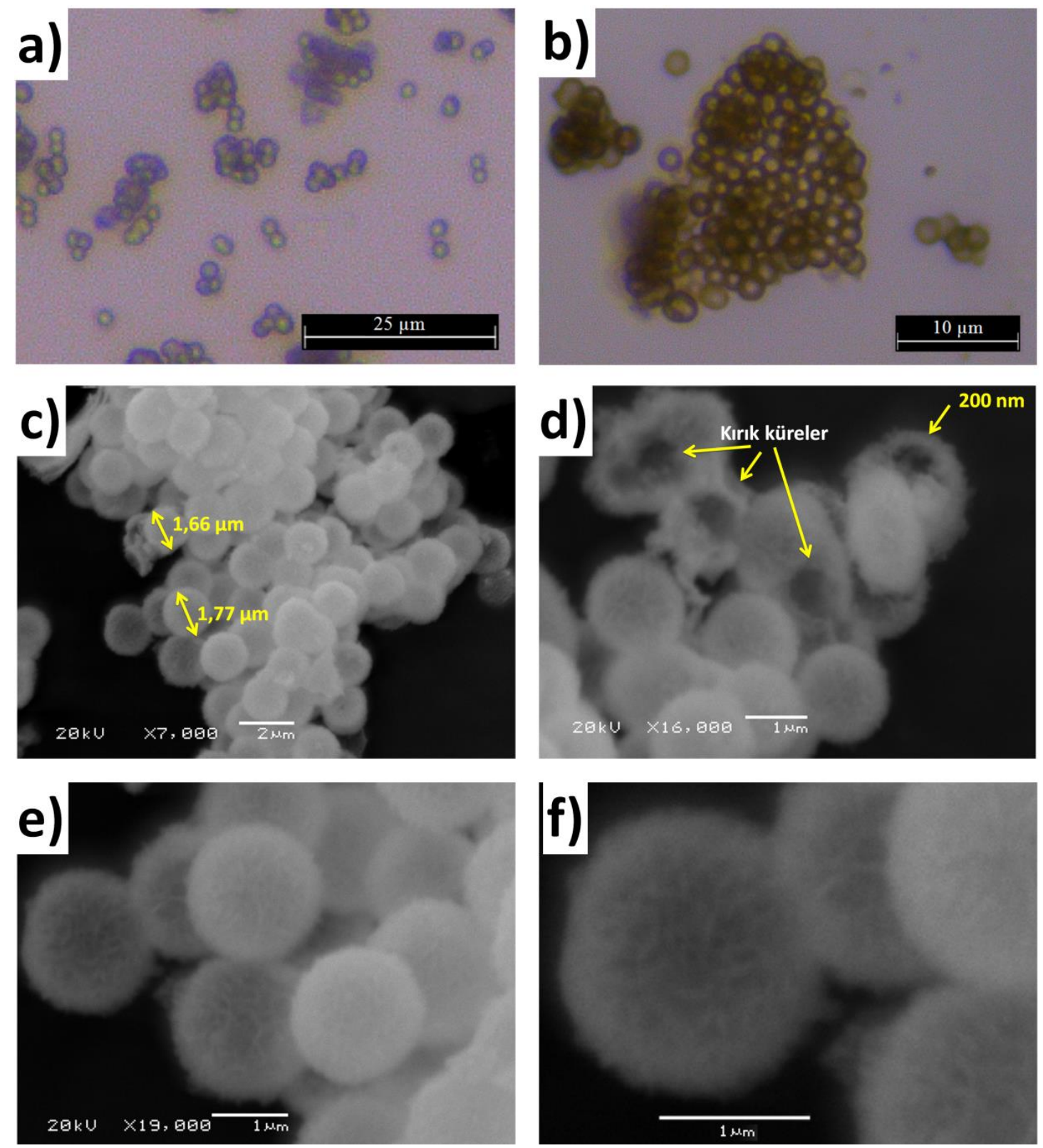

Şekil 2. a) PS kürelerin ve b) kalsinasyon sonrası mangan oksit@nikel oksit içi kovuk kürelerin ışık mikroskop görüntüleri, c-d) kalsinasyon sonrası mangan oksit@nikel oksit içi kovuk kürelerin farklı büyütmedeki SEM görüntüleri. 
SEM görüntüleri incelendiğinde ise 1,60-1,77 $\mu \mathrm{m}$ çapında ve kabuk kalınlığı $200 \mathrm{~nm}$ civarında olan metal oksit kürelerin elde edildi görülmektedir. Şekil 2(f) incelendiğinde metal oksit kürelerin yüzeyinin oldukça pürüzlü olduğu hatta nanolevhaların varlığı görülmektedir. Bu yüksek pürüzlü hal kataliz uygulamalar için önemli avantajlar sunabilecek türdedir. Elde edilen metal oksit kabuk kalınlığının kürelerin stabil olarak kalması için yeterli seviyede olduğu da az sayıda kırık kürelerin varlığından anlaşılmaktadır.

XRD ölçümlerinde ise, PS kürelerin ilk tabakasını oluşturan az miktardaki mangan oksit öncüsü bileşiği ile ikinci tabakadaki nikel oksit öncü bileşiği reaksiyona girerek \%1,3 oranında mangan oksit türünde bir tabaka oluşturduğu belirlendi. i̇kinci tabakanın ise, \% 98,7 oranında nikel oksit oluşturduğu belirlendi (Şekil 3). XRD ölçümü $(\% 1,3)$ ve TGA ölçümünden $(\% 1,56)$ görüleceği gibi ilk tabakanın kalınlığı neredeyse yok denecek kadar düşük seviyededir. Mangan oksit olduğu düşünülen fakat tam olarak içeriği aydınlatılamayan bu fazın iki tabakanın kaynaşma ürünü mangan nikel oksit ve mangan oksit fazlarının karışımı şeklinde olacağı düşünülmektedir. Özellikle $600{ }^{\circ} \mathrm{C}^{\prime}$ de 1 saat tutularak kalsinasyon işleminin tamamlanması ve daha uzun süre tutulmayarak içi kovuk kürelerin birbiri ile kaynaşmasının önüne geçilmeye çalışılmıştır (Qian et al. 2007, Tong et al. 2012, D. B. Wang et al. 2005, Wu et al. 2007, Zhang et al. 2012).

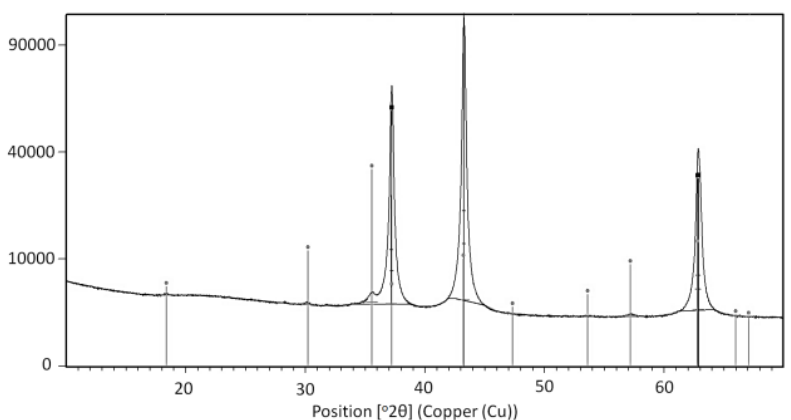

Şekil 3. Mangan oksit@nikel oksit içi kovuk kürelerin XRD analizi.

\section{Sonuç}

Kontrollü çöktürme tekniği ile 1,60-1,77 $\mu \mathrm{m}$ çapında ve $200 \mathrm{~nm}$ kabuk kalınlığında içi kovuk çift tabakalı mangan oksit@nikel oksit kürelerin üretimi gerçekleştirildi. İçi kovuk kürelerin dış tabakasını oluşturan nikel oksit'in nano-levhalar şeklinde kataliz çalışmaların önemli avantaj sunan oldukça pürüzlü bir dış yüzeye sahiptir. Sert şablon kullanımı bakımından yeni bir yaklaşım sunan bu çalışma da, sert şablon olarak PDEA- $b$-PDMA blok kopolimeri ile stabilize edilmiş PS küreler kullanıldı. Üretilen PS kürelerin yüzeyi PDMA saçakları (bloğu) ile sarılıdır ve bu onlara inorganik türleri daha iyi adsorplama ve stabilize etme yeteneğini kazandırır. Bu türdeki sert şablonların, yüzeyi modifiye edilmiş PS kürelere göre daha fazla fonksiyonel gruplara sahip olması ve küresel PS fırçalara göre ise daha basit bir teknikle hazırlanıyor olması gibi önemli avantajları vardır. Bu çalışma ve üretimine devam edilen diğer inorganik içi kovuk kürelerin ilerleyen zamanlarda kataliz çalışmalarında kullanılması oldukça muhtemeldir.

\section{Teşekkür}

PDEA- $b$-PDMA blok kopolimerini temin ettiğim ve bazı analizlerin yapılmasına imkan sağlayan Eskişehir Osmangazi Üniversitesi (ESOGÜ) Kimya Bölümü Öğretim Üyesi Prof. Dr. Vural BÜTÜN'e teşekkürlerimi borç bilirim. XRD analizleri için ESOGÜ Merkezi Araştırma Laboratuvarı Uygulama ve Araştırma Merkez'inde (ARUM) görevli Doç. Dr. Gökhan DiKMEN'e ayrıca teşekkür ederim.

\section{Kaynaklar}

Abdullah, J. A., Al Lafi, A. G., Amin, Y. and Alnama, T., 2018. A Styrofoam-nano manganese oxide based composite: Preparation and application for the treatment of wastewater. Applied Radiation and Isotopes, 136, 73-81.

Amalvy, J. I., Unali, G. F., Li, Y., Granger-Bevan, S., Armes, S. P., Binks, B. P., Rodrigues, J. A. and Whitby, C. P., 2004. Synthesis of sterically stabilized polystyrene latex particles using cationic block copolymers and macromonomers and their application as stimulusresponsive particulate emulsifiers for oil-in-water emulsions. Langmuir, 20, 11, 4345-4354. 
Amari, H., Guerrouache, M., Mahouche-Chergui, S., Abderrahim, R. and Carbonnier, B., 2017. 2Aminothiazole-functionalized triazine-modified polystyrene decorated with gold nanoparticles as composite catalyst for the reduction of 4nitrophenol. Reactive \& Functional Polymers, 121, 58-66.

Bousquet, A., Ibarboure, E., Heroguez, V., Papon, E., Labrugere, C. and Rodriguez-Hernandez, J., 2010. Single-step process to produce functionalized multiresponsive polymeric particles. Journal of Polymer Science Part a-Polymer Chemistry, 48, 16, 3523-3533.

Bütün, V., Kaynak, B., Esenoğlu, E. and Gül, G., 2004. Tersiyer amin merakrilatlara dayalı suda çözünebilen kopolimerlerin sentezi, seçimli kuaternizasyonu ve stabilizör olarak lateks sentezinde kullanılması. e Polimer işleme ve Geri Kazanımı Sempozyumu ve Sergisi (POLYSEM2004), Mersin.

Cai, W. J., Wang, W. Q., Yang, Y. Q., Ren, G. H. and Chen, T., 2014. Sulfonated polystyrene spheres as template for fabricating hollow compact silver spheres via silver-mirror reaction at low temperature. Rsc Advances, 4, 5, 2295-2299.

Deng, Z. W., Chen, M., Gu, G. X. and Wu, L. M., 2008. A facile method to fabricate $\mathrm{ZnO}$ hollow spheres and their photocatalytic property. Journal of Physical Chemistry $B, 112,1,16-22$.

Fei, J. B., Cui, Y., Yan, X. H., Qi, W., Yang, Y., Wang, K. W., He, Q. and Li, J. B., 2008. Controlled preparation of $\mathrm{MnO} 2$ hierarchical hollow nanostructures and their application in water treatment. Advanced Materials, 20, 3, 452-+.

Gawande, M. B., Pandey, R. K. and Jayaram, R. V., 2012. Role of mixed metal oxides in catalysis scienceversatile applications in organic synthesis. Catalysis Science \& Technology, 2, 6, 1113-1125.

Han, X., Zhou, L. H., Liu, H. L. and Hu, Y., 2007. Effect of in situ oxidization with potassium permanganate on the morphologies of SEBS membranes. Polymer Degradation and Stability, 92, 1, 75-85.

Hu, J., Chen, M., Fang, X. S. and Wu, L. W., 2011. Fabrication and application of inorganic hollow spheres. Chemical Society Reviews, 40, 11, 54725491.

Huang, S. B., Yu, X. J., Dong, Y. M., Li, L. and Guo, X. H., 2012. Spherical polyelectrolyte brushes: Ideal templates for preparing $\mathrm{pH}$-sensitive core-shell and hollow silica nanoparticles. Colloids and Surfaces aPhysicochemical and Engineering Aspects, 415, 2230.

Itoh, T., Abe, I., Tamamitsu, T., Shimomoto, H., Inoue, K. and Ihara, E., 2014. Surface structure of stimuliresponsive polystyrene particles prepared by dispersion polymerization with a polystyrene/poly( Llysine) block copolymer as a stabilizer. Polymer, 55, 16, 3961-3969.

Kawahashi, N., Persson, C. and Matijevic, E., 1991. Zirconium compounds as coatings on polystyrene latex and as hollow spheres. Journal of Materials Chemistry, 1, 4, 577-582.

Kawahashi, N. and Shiho, H., 2000. Copper and copper compounds as coatings on polystyrene particles and as hollow spheres. Journal of Materials Chemistry, 10, 10, 2294-2297.

Li, B. X., Rong, G. X., Xie, Y., Huang, L. F. and Feng, C. Q., 2006. Low-temperature synthesis of alpha-MnO2 hollow urchins and their application in rechargeable $\mathrm{Li}+$ batteries. Inorganic Chemistry, 45, 16, 6404-6410.

Lu, Y., Hoffmann, M., Yelamanchili, R. S., Terrenoire, A., Schrinner, M., Drechsler, M., Moller, M. W., Breu, J. and Ballauff, M., 2009. Well-defined crystalline TiO2 nanoparticles generated and immobilized on a colloidal nanoreactor. Macromolecular Chemistry and Physics, 210, 5, 377-386.

Munoz-Bonilla, A., van Herk, A. M. and Heuts, J. P. A., 2010. Adding stimuli-responsive extensions to antifouling hairy particles. Polymer Chemistry, 1, 5, 624-627.

Nie, G., Li, G., Wang, L. and Zhang, X., 2016. Nanocomposites of polymer brush and inorganic nanoparticles: preparation, characterization and application. Polymer Chemistry, 7, 4, 753-769.

Ohnishi, M., Kozuka, Y., Ye, Q. L., Yoshikawa, H., Awaga, K., Matsuno, R., Kobayashi, M., Takahara, A., Yokoyama, T., Bandow, S. and lijima, S., 2006. Phase 
selective preparations and surface modifications of spherical hollow nanomagnets. Journal of Materials Chemistry, 16, 31, 3215-3220.

Pal, J. and Pal, T., 2015. Faceted metal and metal oxide nanoparticles: design, fabrication and catalysis. Nanoscale, 7, 34, 14159-14190.

Polzer, F., Holub-Krappe, E., Rossner, H., Erko, A., Kirmse, H., Plamper, F., Schmalz, A., Muller, A. H. E. and Ballauff, M., 2013. Structural analysis of colloidal MnOx composites. Colloid and Polymer Science, 291, 3, 469-481.

Qian, H. S., Lin, G. F., Zhang, Y. X., Gunawan, P. and Xu, R., 2007. A new approach to synthesize uniform metal oxide hollow nanospheres via controlled precipitation. Nanotechnology, 18, 35.

Reis, B. M., Armes, S. P., Fujii, S. and Biggs, S., 2010. Characterisation of the dispersion stability of a stimulus responsive core-shell colloidal latex. Colloids and Surfaces a-Physicochemical and Engineering Aspects, 353, 2-3, 210-215.

Sun, X. M., Liu, J. F. and Li, Y. D., 2006. Use of carbonaceous polysaccharide microspheres as templates for fabricating metal oxide hollow spheres. Chemistry-a European Journal, 12, 7, 20392047.

Tong, G. X., Liu, F. T., Wu, W. H., Shen, J. P., Hu, X. and Liang, Y., 2012. Polymorphous alpha- and beta$\mathrm{Ni}(\mathrm{OH})(2)$ complex architectures: morphological and phasal evolution mechanisms and enhanced catalytic activity as non-enzymatic glucose sensors. Crystengcomm, 14, 18, 5963-5973.

Wang, D. B., Song, C. X., Hu, Z. S. and Fu, X., 2005. Fabrication of hollow spheres and thin films of nickel hydroxide and nickel oxide with hierarchical structures. Journal of Physical Chemistry B, 109, 3, 1125-1129.

Wang, X. J., Feng, J., Bai, Y. C., Zhang, Q. and Yin, Y. D., 2016. Synthesis, properties, and applications of hollow micro-/nanostructures. Chemical Reviews, 116, 18, 10983-11060.

Watanabe, M., Aritomo, H., Yamaguchi, I., Shinagawa, T., Tamai, T., Tasaka, A. and Izaki, M., 2007. Selective preparation of zinc oxide nanostructures by electrodeposition on the templates of surfacefunctionalized polymer particles. Chemistry Letters, $36,5,680-681$.

Wu, X. F., Chen, Y. F., Li, Q. Y. and Wei, L. Q., 2007. Preparation and characterization of integral hollow microspheres of nickel hydroxide and nickel oxide. Nanoscience and Technology, Pts 1 and 2, 121-123, $187-190$

Yang, L. L., Kong, X. W., Wang, J., Pan, M. Q., Yang, W. Q., Yang, J. H. and Jiang, W. L., 2016. Synthesis and photocatalytic performance of $\mathrm{ZnO}$ hollow spheres and porous nanosheets. Journal of Materials ScienceMaterials in Electronics, 27, 1, 203-209.

Ye, Q. L., Yoshikawa, H. and Awaga, K., 2010. Magnetic and Optical properties of submicron-size hollow spheres. Materials, 3, 2, 1244-1268.

Yoon, S., 2014. Facile microwave synthesis of CoFe2O4 spheres and their application as an anode for lithium-ion batteries. Journal of Applied Electrochemistry, 44, 9, 1069-1074.

Zhang, P. P., Ma, X. M., Guo, Y. M., Cheng, Q. Q. and Yang, L., 2012. Size-controlled synthesis of hierarchical $\mathrm{NiO}$ hollow microspheres and the adsorption for Congo red in water. Chemical Engineering Journal, 189, 188-195.

Zhu, Y., Chen, K. M., Wang, X. and Guo, X. H., 2012. Spherical polyelectrolyte brushes as a nanoreactor for synthesis of ultrafine magnetic nanoparticles. Nanotechnology, 23, 26. 